\title{
Simultaneous nitrite/nitrate imagery at millimeter scale through the water-sediment interface
}

\author{
Metzger Edouard ${ }^{1,{ }^{*}}$, Thibault Aubin ${ }^{1,2}$, Cesbron Florian ${ }^{1,3}$, Barbe Anthony ${ }^{1}$, Launeau Patrick ${ }^{4}$, \\ Jezequel Didier ${ }^{5}$, Mouret Aurélia ${ }^{1}$
}

${ }^{1}$ Université d'Angers, LPG-BIAF, UMR CNRS 6112, 49045 Angers Cedex, France

${ }^{2}$ Ifremer, LBCM, Rue de l'lle d'Yeu, 44300 Nantes, France

${ }^{3}$ University of West Florida, CEDB, 11000 University Parkway, Pensacola, FL 32514 ,USA

${ }^{4}$ Université de Nantes, LPG-N, UMR CNRS 6112, 44322 Nantes, France

${ }^{5}$ Institut de Physique du Globe de Paris, Sorbonne Paris Cité, Univ. Paris Diderot, UMR 7154 CNRS,

75005 Paris, France

${ }^{*}$ Corresponding author: Edouard Metzger, +33(0)2 417353 82. ; Fax: +33(0)2 417353 52. ;

email address : edouard.metzger@univangers.fr

\begin{abstract}
:
The present study describes new procedures to obtain at millimeter resolution the spatial distribution of nitrite and nitrate in porewaters, combining diffusive equilibrium in thin films (DET), colorimetry and hyperspectral imagery. Nitrite distribution can be easily achieved by adapting the well-known colorimetric method from Griess (1879) and using a common flatbed scanner with a limit of detection about $1.7 \mu \mathrm{mol}$ L-1. Nitrate distribution can be obtained after reduction into nitrite by a vanadium chloride reagent. However, the concentration of vanadium chloride used in this protocol brings coloration with a wide spectral signature that creates interference only deconvolvable by imaging treatment from an entire visible spectrum for each pixel (spectral analysis). This can be achieved by hyperspectral imaging. The protocol retained in the present study allows obtaining a nitrite/nitrate image with micromolar limit of detection. The methods were applied in sediments from the Loire Estuary after different treatments and allowed to precisely describe two-dimensional millimeter features. The present technique adds to the combination of gel-colorimetry and hyperspectral imagery a very promising new application of wide interest for environmental issues in the context of early diagenesis and benthic fluxes.
\end{abstract}




\section{Graphical abstract :}

High-resolution 2D speciation of $\mathrm{NO}_{x}^{-}$in a estuarine mudflat
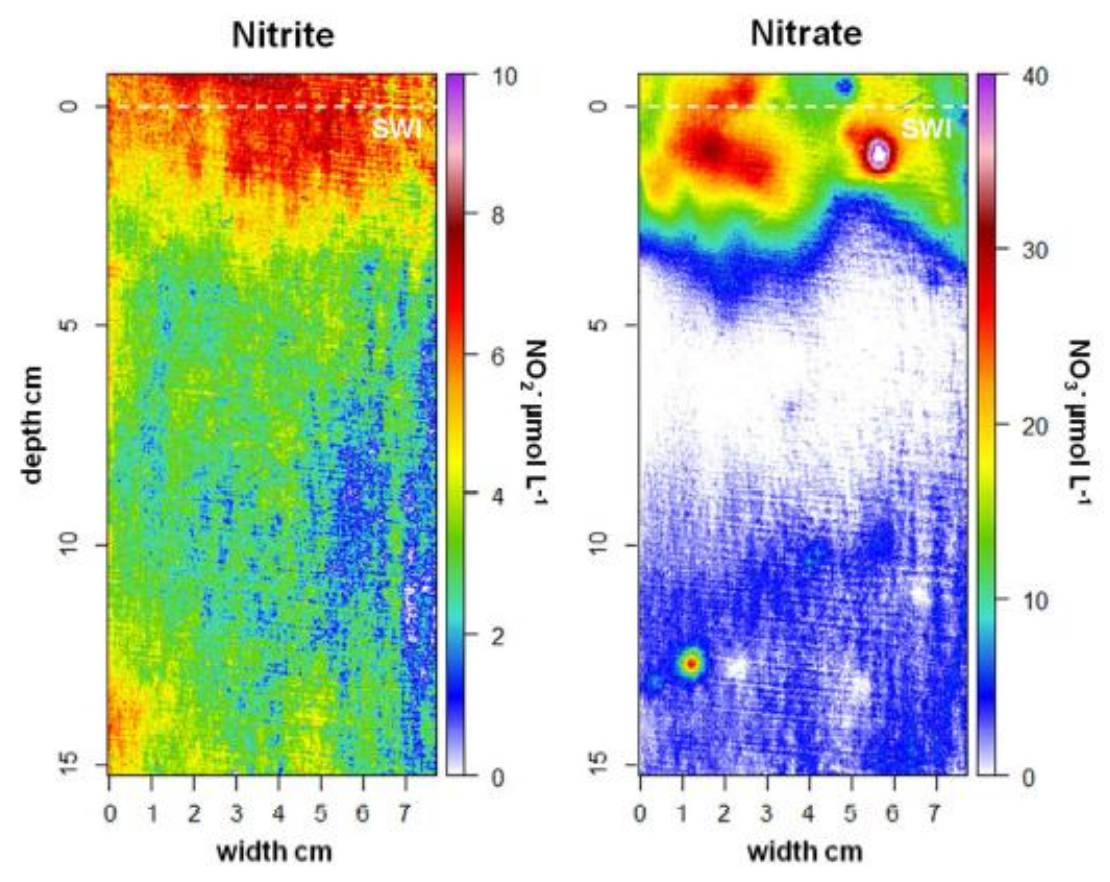


\section{Introduction}

Combination of diffusive equilibrium in thin film gel techniques (DET), ${ }^{1}$ and spectrophotometry allows theoretically to reach speciation of nutrients, completing the set of tools existing for porewater chemistry at millimeter and sub-millimeter resolution. However, the main limitation of the combination of colorimetry and gel techniques is the limit of detection (LOD) induced by very small samples (below $100 \mu \mathrm{L}$ ) and subsequent dilution that is required to get sufficient volume for analytical purposes. ${ }^{2-4}$ Only miniaturization and sensibility increase of analytical devices permit to overcome this limitation (e.g.: Nanodrop coupled with microplates). This is why most studies combine DET gel sampling with total elementary analyses such as ICP-MS or GF-AAS. ${ }^{5}$ Few studies have combined DET sampling with ion chromatography to determine sulfate and nitrate concentrations. ${ }^{6-8}$ In these works, the gel of the DET probe was cut into strips corresponding to a resolution of 2 to 20 $\mathrm{mm}$. This generated at lot of samples to process and represents a time-consuming method, to finally obtain a $1 \mathrm{D}$ profile with a rather low resolution. Another alternative to remove such limitation was to generate an image of the distribution of dissolved compounds within the gel by direct contact of the DET gel with a reagent, either contained in another reactive gel ${ }^{9,10}$ or in a solution. ${ }^{11}$ Although simple and fast this approach has an important limitation in the case of slow color development: (1) a rapid and uncontrolled back-diffusion of chemicals into the reagent gel or solution, before coloring reaction, combined with lateral diffusion within the probe gel, that forbids any modeling for recalculation of 2D features using kinetics-transport modeling. ${ }^{9,10}$ Such limitation is less important for fast-kinetic reactions such as for iron and alkalinity, which develop coloration in a short time $(<1 \mathrm{~min}$.$) ; (2) a need for a rapid$ processing that generates important handling, forbids postponing analysis and limits the number of probes to be processed. Recent studies ${ }^{12,13}$ showed the possibility to freeze gel probes allowing further analysis and almost no limit to the number of probe deployment. However, the major input of these studies was the use of a hyperspectral camera allowing a 
better sensitivity and a higher spectral resolution (few nanometers instead of few hundred from a RGB image). A spectrum for each pixel allows different post acquisition treatments allowing separation of different contributors to the image at a pixel scale. Cesbron et al. ${ }^{12}$ proposed a method to simultaneously analyze dissolved iron and reactive phosphorous. This is an excellent alternative to probes built with different layers of gel, ${ }^{11}$ each one being dedicated to one chemistry that need handling skills and do not guarantee a perfect match of the different images (i.e.: distortion of the gel, gap between images).

As mentioned above, nitrate profiles obtained with gel techniques are limited by LOD of ion chromatography and elution of gel into a solution that significantly dilutes chemicals. For this reason, nitrite is rarely detected and only nitrate quantification can be done. The development of a nitrate probe as a microelectrode by a Danish group in early $2000,{ }^{14,15}$ brought an alternative to perform high-resolution profiling within the sediment at high-resolution (ca.; $100 \mu \mathrm{m})$. Despite an attempt of commercialization by Unisense, only researchers from the original group were able to perform such profiles. Achieving high-resolution profiles of nitrite and nitrate resolution, as shown by several authors, is of major importance to quantify benthic diffusive fluxes and to investigate new reactional pathways for nitrogen transformations within the sediment that is affected by bioturbation. However, at the scale of a microenvironment (burrow wall, root apex, etc...), only multi-species high-resolution profiles and/or 2D distribution can achieve correlations between chemical species despite high spatial heterogeneity. The recent publication of a method of nitrate reduction using a solution of vanadium chloride ${ }^{16,17}$ allow us to examine the possibility to propose a colorimetric protocol for simultaneous nitrite and nitrate determination combining gel sampling, colorimetry and a 2 dimensional image acquisition. Firstly, the present study aims to transpose the famous Griess reaction ${ }^{18}$ to gel technique in order to obtain a nitrite image of porewater in a sedimentary setting at a sub-millimeter resolution. Secondly, we examine the possibility to 
73 revisit the procedure using vanadium chloride as a nitrate reducer in order to achieve a 74 nitrite/nitrate 2D probe that could successfully be deployed within the sediment.

\section{2. Experimental section}

\subsection{Principle of the method}

77 A polyacrylamide gel probe is prepared (polymerized, rinsed and degassed) and deployed within the sediment until diffusional equilibrium with porewater solutes (including $\mathrm{NO}_{2}{ }^{-}$and $\left.\mathrm{NO}_{3}{ }^{-}\right)$is reached.

A first reagent gel is prepared, containing the Griess ${ }^{18,19}$ reagent: the coloring reagent gel, allowing specifically the nitrite determination. A second reagent gel is prepared using vanadium chloride: the reducing reagent gel that reduces nitrate into nitrite, allowing nitrate determination. The probe gel is laid down onto the first coloring reagent gel in order to obtain an image of nitrite distribution as variations of pink coloration over the gel assemblage.

An imagery device allows the digitalization of the colored image (flatbed scanner for nitrite imagery alone, or hyperspectral camera for both nitrite and nitrate determinations).

87 In order to obtain the nitrate distribution over the gel, the reducing reagent gel is added to the two-layer assemblage after the first digitalization for nitrite. A second digitalization is operated on the three-layer assemblage and the subtraction of signals between both images is performed for each pixel in order to obtain the nitrate contribution over the gel probe.

\subsection{Sampling gel preparation and deployment} high, $150 \mathrm{~mm}$ wide and $3 \mathrm{~mm}$ thick). The plate has a central depression of $1 \mathrm{~mm}$ depth $(180 \times$ $97 \mathrm{~mm}$, length $\times$ width) that holds the hydrogel keeping the probe tight. ${ }^{12}$ The gel is maintained and protected from sediment by a PVDF hydrophilic membrane $(0.2 \mu \mathrm{m}$, 
97 gel is a polyacrylamide DET gel prepared according to Jézéquel et al., ${ }^{9}$ adapted from Zhang 98 and Davison. ${ }^{20}$ In brief, a mixture of acrylamide (30\%, Roth), bisacrylamide (2\%, Roth), 99 TEMED (Roth) as a catalyzer and ammonium persulfate (APS; 1\%, Sigma-Aldrich) as a 100 polymerization initiator, is cast between two plates separated by a spacer. Polymerization 101 occurred after ca. $15 \mathrm{~min}$. at room temperature. The gel is rinsed and stored in deionized 102 water (Millipore Milli- $\mathrm{Q}^{\circledR}$ system) until use. Before hydration, thickness of the gel is $0.92 \mathrm{~mm}$ 103 but reaches about $1 \mathrm{~mm}$ after $1 \mathrm{~h}$ hydration. In order to avoid oxygen contamination of anoxic 104 sediment during probe deployment, the probe is deoxygenated by nitrogen bubbling in 105 deionized water (Millipore Milli- $\mathrm{Q}^{\circledR}$ system) at least $5 \mathrm{~h}$ before deployment. Probes were 106 deployed into sediment for 5 hours allowing equilibration. ${ }^{11}$

\subsection{Reagent gel preparation}

Reagent gels are $0.46 \mathrm{~mm}$-thick $(0.5 \mathrm{~mm}$ after hydration), polyacrylamide hydrogels with the same composition as probe gels. ${ }^{20}$ After polymerization, reagent gels are equilibrated during at least $30 \mathrm{~min}$ with $50 \mathrm{~mL}$ of a reagent solution in a plastic bag. The

111 coloring reagent solution contains $\quad 0.07 \%$ sulfanilamide (Roth), $\quad 0.01 \quad \% \quad$ N-1112 naphthylethylenediamine dihydrochloride (NEDD) (Roth) and $0.14 \% \mathrm{HCl}$ (Roth) in Milli-

$113 \mathrm{Q}{ }^{\circledR}$ water (uncolored solution). The reducing reagent gel is equilibrated with $50 \mathrm{~mL}$ of a 114 vanadium chloride solution $\left(\mathrm{VCl}_{3}\right.$ (Sigma Aldrich) $2.9 \%(\mathrm{w} / \mathrm{v}), \mathrm{HCl} 1.8 \%(\mathrm{w} / \mathrm{v})$ from a $30 \%$ 115 suprapur acid (Roth)). This gel is colored green by the vanadium salt.

\subsection{Standard gel preparation}

117 The standard gel is prepared by diffusion of standard solutions into a 1-mm thick gel similar

118 to the probe gel: ${ }^{12}$ the gel is placed onto a Plexiglas ${ }^{\circledR}$ plate and covered by a second 119 Plexiglas ${ }^{\circledR}$ plate with 7 circular wells $(2.1 \mathrm{~cm}$ i.d.) drilled into it. Each well is equipped with a 120 cylindrical tube representing a $5 \mathrm{~mL}$ vial when put onto the gel (the base of each cylinder 
121 overhangs slightly from the Plexiglas ${ }^{\circledR}$ plate in order to be pressed onto the gel). The

122 Plexiglas ${ }^{\circledR}$ plates are tightened together with 8 small clamps, ensuring sufficient pressure on

123 the gel to avoid leakage of standard solutions. A volume of $3.5 \mathrm{~mL}$ of each standard solution

124 was poured into a well and incubated during $1 \mathrm{~h}$ in order to ensure diffusive equilibration.

125 Nitrate and nitrite standard solutions were prepared from $\mathrm{NaNO}_{3}$ and $\mathrm{NaNO}_{2}$ salts 126 respectively (Fluka).

128 After equilibration, the coloring reagent gel is removed from the bag and quickly drained

129 before being laid down onto a white plate. Residual drops are gently wiped and the probe gel

130 is laid onto it. The whole assemblage is covered by a cellulose acetate film that protects

131 against evaporation, then scanned after $15 \mathrm{~min}$, the necessary time for the NEDD to react with

132 nitrite and form the so-called pink Azo dye revealing the 2D nitrite distribution.

133 To reduce nitrate, the cellulose acetate film is carefully removed from the double layer gel

134 assemblage (i.e., probe + nitrite reagent gels) and a nitrate reducing gel is laid on top of it.

135 The three-layer gel assemblage is then covered by a clean cellulose acetate film and incubated

136 for $20 \mathrm{~min}$ in a $50^{\circ} \mathrm{C}$ oven under water-saturated atmosphere. This heating step is crucial for

137 accelerating the kinetics of nitrate reduction. At that point, the produced nitrite has reacted

138 with the Griess reagent and the three-layer gel can be scanned at its turn. The calibration gel is

139 processed like the in situ gel probe.

140 Two calibration gels (one from nitrate solutions and one from nitrite solutions) are necessary

141 for estimating the efficiency of nitrate reduction. In order to save time and guarantee

142 comparable conditions, both nitrite and nitrate calibration gels (two strips) are laid side-by-

143 side onto the same reagent gels to be processed together. We also recommend preparing a

144 solution with both nitrate and nitrite for quality control. Solutions ranged between 1.5 and 40

$145 \mu \mathrm{mol} \mathrm{L}{ }^{-1}$ in either $\mathrm{NO}_{2}{ }^{-}$or $\mathrm{NO}_{3}{ }^{-}$. Linear range of the color intensity towards concentration can 
146 be extended using thinner probe or standard gels (e.g., 0.5 instead of $1.0 \mathrm{~mm}$ ). Hence, thinner 147 gels reduce the optical path, resulting in weaker color intensities. As a consequence, the 148 saturation concentration increases but LOD increases as well.

\subsection{D imagery methods}

\subsubsection{Nitrite imagery from commercial flatbed scanner}

To obtain only nitrite distribution, a commercial flatbed scanner (Canon Canoscan LiDE $600 \mathrm{~F})$ was used. From scanned images, intensity of colored zones of the 2D probe was processed by Image ${ }^{\circledR}$ software. Images were decomposed into primary color intensities (red, green and blue (RGB), at about $100 \mathrm{~nm}$ wavelength resolution), each being converted to a gray-scale image ${ }^{9}$. The green color intensity was found to give the most sensitive response, since the nitrite-reagent compound (Azo dye) is pink.

\subsubsection{Hyperspectral data acquisition and treatment for nitrite/nitrate mapping}

This procedure does not require any specific equipment but does not allow simultaneous quantification of nitrite and nitrate because of the dark green coloration of the reducing reagent and its spectral signature prevents the pink Azo dye to be read. When simultaneous nitrate and nitrite distributions are sought, both nitrite and nitrite+nitrate images have to be obtained with a hyperspectral camera (here, a HySpex VNIR 1600) that has a sufficient wavelength resolution to separate signals coming from different layers of colored gel. The camera has 160 channels, covering the spectral range from $400 \mathrm{~nm}$ to $900 \mathrm{~nm}$, with a spectral resolution of $4.5 \mathrm{~nm}$ and a sampling interval of $3.7 \mathrm{~nm}$ allowing the analysis of a continuing spectrum. The acquisition time was 2 minutes. The flat assembly gel probe + reactive complex + transparent film was laid down on a Spectralon ${ }^{\circledR}$ plate and then scanned in front of the camera and under controlled halogen light source. The camera was set up in the laboratory 
169 to scan samples with square pixels providing a spatial resolution of about $190 \times 190 \mu \mathrm{m}$ per 170 pixel (camera about $1 \mathrm{~m}$ above samples).

171 According to Cesbron et al., ${ }^{12}$ reflectance spectra results of an intimate mixing of colored 172 reagents within a transparent gel. Therefore it is possible to decompose each pixel into 173 different end-members according to their spectra. The decomposition of each pixel is

174 calculated as a linear combination of the logarithm of the different end-member spectra using 175 ENVI ${ }^{\circledR}$ software (unmixing function). In the present study, 3 end-member spectra are 176 considered: (1) the spectral background which corresponds to a Spectralon ${ }^{\circledR}$ plate + the two177 or three-layer 2D gel (for respectively the first and the second scan); (2) a spectral nitrite end178 member from the highest nitrite concentration of the calibration gel and (3) the spectrum 179 corresponding to the vanadium(III) green coloration obtained from a nitrite-free part of the 180 calibration gel. This unmixing procedure is applied to the calibration gel for signal linearity 181 checking as concentration increases and to the probe gel for $\mathrm{NO}_{2}{ }^{-}$and $\mathrm{VCl}_{3}$ signals separation.

\subsection{Validation of the method}

\subsubsection{Validation of nitrite/nitrate separation}

184 To ensure a complete separation of signal from nitrite and from nitrate, calibration standards

185 were performed with two composite standards containing both species. The double standard 186 gel (made of two strips, one for each chemical species) is laid onto the Griess reagent gel. At

187 the first stage of the treatment, only the set of circles corresponding to the nitrite standard gel

188 is supposed to turn pink. Obviously, the nitrite + nitrate composite circle would become as 189 pink as its corresponding nitrite concentration enables it.

190 Once the assemblage is scanned by the hyperspectral camera, the $\mathrm{VCl}_{3}$ reducing reagent gel is

191 added and the new assemblage goes into the oven as described above (section 2.5). After 20

192 minutes, a new scanning is performed and reduced nitrate into nitrite appears pink. It is 
193

194

195

196

197

198

199

214 The quantification of nitrate on the probe gel (three-layer gel) will be for each pixel:

215

216 217

important to note that the $\mathrm{VCl}_{3}$ reducing reagent gel is dark green and low intensity pink is not visible with naked eyes.

After unmixing calculation (section 2.4.), nitrite reflectance from both images can be achieved (i.e. before and after addition of the dark green $\mathrm{VCl}_{3}$ reducing agent gel). From the second image (with the $\mathrm{VCl}_{3}$ reducing reagent gel) a nitrite + newly produced nitrite image is obtained. From the literature, ${ }^{16}$ it is known that 20 min at $50^{\circ} \mathrm{C}$ is too short to achieve a complete reduction but a longer incubation time will affect the distribution of nitrate because of lateral diffusion (i.e., there is a competition between reduction kinetics and loss of signal by diffusion, see section 3.1. and 3.2.). The comparison of standard curve slopes between nitrite and nitrate standards allows quantifying the efficiency of nitrate reduction into nitrite for each set of experiments and therefore calculation of nitrate concentration.

Summarizing, the first image is used for direct nitrite standardization while the second is used to quantify nitrate reduction efficiency and therefore to quantify the sum of nitrite and the part of nitrate that was reduced during the experience. The same two-step procedure is applied to the probe gel.

The quantification of nitrite on the probe gel (two-layer gel) is for each pixel:

$\left[\mathrm{NO}_{2}^{-}\right]=\left[\left(\right.\right.$slope $\left._{\text {nitrite }} \times \mathrm{R}_{\text {nitrite }}\right)$

where slope nitrite $_{\text {is }}$ the slope of the nitrite standard curve of the first image (without $\mathrm{VCl}_{3}$ gel);

$\mathrm{R}_{\text {nitrite }}$ is the reflectance of the assembled probe gel before addition of the $\mathrm{VCl}_{3}$ gel.

$\left[\mathrm{NO}_{3}{ }^{-}\right]=\left[\left(\right.\right.$slope $\left.\left._{\text {nitrite }}{ }^{\mathrm{VCl}} \times \mathrm{R}_{\mathrm{VCl} 3}\right)-\left[\mathrm{NO}_{2}{ }^{-}\right]\right] \times$slope $_{\text {nitrite }} \mathrm{VCl}^{\mathrm{V}} /$ slope $_{\text {nitrate }} \mathrm{VCl3}$ 
218 where slope nitrite $^{\mathrm{VCl}}$ is the slope of the nitrite standard curve of the second image (with $\mathrm{VCl}_{3}$

219 gel); $\mathrm{R}_{\mathrm{VCl}}$ is the reflectance of the assembled probe gel after addition of the $\mathrm{VCl}_{3}$ gel;

220 slope $_{\text {nitrate }}{ }^{\mathrm{VCl} 3}$ is the slope of the nitrate standard curve; $\left[\mathrm{NO}_{2}{ }^{-}\right]$is the concentration of nitrite

221 for each pixel after standardization of the assembled probe gel before addition of the $\mathrm{VCl}_{3}$ gel.

\subsubsection{Validation of the method for sediment}

223 A series of laboratory experiments using estuarine sediment was realized in order to test the 224 nitrite/nitrate method on more realistic conditions. Sediment was sampled in the Brillantes 225 mudflat from the River Loire estuary $\left(47^{\circ} 16^{\prime} 56.00^{\prime \prime} \mathrm{N} \quad 2^{\circ} 3^{\prime} 47.00^{\prime \prime} \mathrm{W}\right)$. The sediment is mainly 226 composed of silt (92\%) with some clay (6\%) and sand (2\%) and is colonized in particular 227 by microphytobenthic films (diatoms up to $60 \mathrm{mg} \mathrm{m}^{-2} ;{ }^{21}$ and bioturbating macrofauna 228 (mainly Hediste diversicolor and Scrobicularia plana; I. Metais, pers. comm.)

229 Laboratory experiment on homogenized sediment: sediment sampling occurred in June 2014.

230 The sediment was sieved with a mesh of $1 \mathrm{~mm}$ in order to remove macrofauna. After

231 homogenization, sediment was covered with estuarine water and left for equilibration for 12

232 days in the laboratory. Overlying water was constantly aerated and the system kept in the dark

233 at room temperature until gel probes deployment. The day before deployment, a burrow was

234 created using a $50 \mathrm{~mL}$ Falcon ${ }^{\circledR}$ tube and gently removed to keep the artificial burrow, and the 235 probe was inserted in the axis of this artificial structure. Before retrieval of the probe, the 236 surface water was sampled (about $5 \mathrm{~cm}$ above the sediment water interface, SWI) and nitrite 237 was analyzed using the classical colorimetric technique with a spectrophotometer.

238 Laboratory experiment on a non-homogenized sediment: sediment sampling occurred in June 239 2015. This time, the sediment was neither sieved nor homogenized in order to maintain 240 possible natural structures that could generate nitrite or nitrate microenvironments such as 241 fecal pellets, dead organisms or burrows. 
243

244

245

246

247

248

249

250

251

252

253

254

255

256

257

258

259

260

261

262

263

264

265

266

267

\section{Results \& Discussion}

\subsection{Colorimetric measurement of nitrite on a gel}

\subsubsection{Nitrite reaction characteristics}

The first test of a protocol for colorimetric nitrite determination using gels consisted in simply starting from the well-known protocol of Griess, ${ }^{18}$ modified for seawater by Bendschneider and Robinson ${ }^{22}$ and detailed by Strickland and Parsons ${ }^{23}$ and Grasshof et al. ${ }^{24}$, adapting reagent proportions to gels. The superimposition of one coloring reagent gel with a thickness of $c a .0 .5 \mathrm{~mm}$ to a probe gel with a thickness of $1 \mathrm{~mm}$ corresponds in a dilution with a ratio of 1/1.5 for nitrite from the sample gel and $1 / 3$ for the chemicals from the reagent gel. The gels were prepared as described section 2. Figure 1 shows the successive images of the calibration gel versus time (Figure 1A) and the corresponding reflectance obtained with the commercial flatbed scanner (Figure 1B). The concentration of nitrite standards ranged from 1.25 to 40 $\mu \mathrm{mol} \mathrm{L}{ }^{-1}$. Coloration clearly appears after $2 \mathrm{~min}$. However, below $5 \mu \mathrm{mol} \mathrm{L} \mathrm{L}^{-1}$ it appears difficult to distinguish nitrite signal with naked eyes and it is necessary to perform a numerical treatment (e.g. with Image ${ }^{\circledR}$ densitometry software). Once it is done, the differences between the standards and the background are significant down to a concentration of $1.25 \mu \mathrm{mol} \mathrm{L}{ }^{-1}$. The time series lasting over $96 \mathrm{~min}$ indicates that the colorimetric reaction is complete $10 \mathrm{~min}$ after contact between the reagent gel and the calibration gel. This supports the idea that the kinetics of colorimetric reaction is similar to classical spectroscopic measurements and in a gel assemblage (see references above).

Figure $1 \mathrm{C}$ shows the evolution of a coloration profile across the edge of the $19.5 \mu \mathrm{mol} \mathrm{L} \mathrm{L}^{-1}$ nitrite standard-well according to time. The good superimposition of profiles within the timeseries indicates that no diffusion seems to take place laterally after contact between the calibration gel and the reagent gel. In particular, there is no visible relaxation of gradient concentration during the colorimetric reaction. However, the asymptotic diffusive profile 
268 centered at the limit of the well, that held the standard solution, seems to indicate that during

269 the equilibrium step between the standard solutions and the calibration gel, nitrite is free to 270 diffuse laterally beyond the well limits. Similar observations were made for iron and 271 phosphorous previously. ${ }^{12}$ These results indicate that the colored component has a much 272 slower diffusion coefficient than the free nitrite. It also suggests that the time of relaxation of 273 the signal by lateral diffusion of solutes in the gel during handling (i.e. from time of probe 274 retrieving to coloration process), is a crucial parameter that strongly limits the maximal 275 resolution of this method and special care must be taken while interpreting sub-millimeter 2D 276 structures. $^{12,25}$

277 Since the signal does not evolve significantly after 10 minutes, the maximum reflectance 278 intensity can be correlated with standard concentrations to realize a standard curve 279 (coefficient of determination of 0.9972). The standard curve allows calculating a limit of 280 detection (3-fold standard error of the blank) of $1.7 \mu \mathrm{mol} \mathrm{L}^{-1}$ and a limit of quantification 281 (LOQ) about $5 \mu \mathrm{mol} \mathrm{L}{ }^{-1}$ (10-fold standard error of the blank). Accuracy is about $2 \mu \mathrm{mol} \mathrm{L}{ }^{-1}$ 282 within the range from 0 to $40 \mu \mathrm{mol} \mathrm{L}^{-1}$.

\subsubsection{Application on a homogenized sediment}

284 Figure 2 shows the two-dimensional distribution of nitrite obtained after deployment of a gel 285 probe within the sieved sediment that was artificially perturbed. The overall feature of the 286 nitrite distribution seems to follow the shape of the perturbation with concentration up to

$28715 \mu \mathrm{mol} \mathrm{L}{ }^{-1}$. The image shows that nitrite concentrations ranged between 10 and $15 \mu \mathrm{mol} \mathrm{L}{ }^{-1}$ 288 in the first centimeter above the SWI. A water sample taken $5 \mathrm{~cm}$ above (near the air-water 289 interface), and analyzed classically (with a spectrophotometer), showed a concentration of 5.8

$290 \mu \mathrm{mol} \mathrm{L}{ }^{-1}$ suggesting a nitrite flux from the sediment into the overlying water of the aquarium.

291 As expected, below $2 \mathrm{~cm}$ depth (and around the artificial burrow), nitrite is depleted reaching 
292 LOD at $5 \mathrm{~cm}$ depth, likely by denitrification or anammox processes occurring in the sediment $293{ }^{26}$ or abiotically due to lower redox potential.

294 The first goal of this paper was to transpose the classical nitrite colorimetric determination to 295 a 2D gel in order to describe nitrite distribution at high resolution affected by any sedimentary 296 structure. The results shown here indicate that this goal was achieved.

\subsection{Simultaneous nitrite and nitrate $2 D$ analysis}

\subsubsection{Optimization of nitrate reduction kinetics}

299 The recent publications ${ }^{16,17}$ considering the use of a vanadium chloride solution instead of the 300 widely used granular copperized cadmium column for nitrate reduction to nitrite ${ }^{27}$ brought new perspectives for the applicability of a colorimetric technique for nitrate in gels. However, there is a major limitation in the transposition of the protocols established by these studies to

303 gel imagery: the slow kinetics of nitrate reduction into nitrite. Hence, a slow colorimetrical

304 reaction would favor relaxation of strong concentration gradients by diffusion of nitrate

305 before immobilization by colorimetrical reaction. To keep the ability of the 2D DET to catch

306 millimeter features in sediment it is necessary to optimize the kinetics of nitrate reduction 307 with $\mathrm{VCl}_{3}$. Schnetger and Lehners (2014) established that a complete reduction of nitrate into 308 nitrite with $\mathrm{VCl}_{3}$ takes 10 hours at ambient temperature and $40 \mathrm{~min}$ at $45^{\circ} \mathrm{C}$ using a $0.57 \%$ 309 (w/v) $\mathrm{VCl}_{3}$ reagent solution. ${ }^{16}$ Similar results were found by Garcia-Robledo et al. (2014). In 310 addition, García-Robledo et al. showed that kinetics is also dependent of the concentration of 311 vanadium and of the acidity of the reducing reagent. ${ }^{17}$ At $50^{\circ} \mathrm{C}$, they showed that time of

312 nitrate reduction decreases by more than a half when $\mathrm{VCl}_{3}$ increases from 0.5 to $2 \%(\mathrm{w} / \mathrm{v})$. A 313 similar experiment was realized in the present study (see supporting information, SI-2) that 314 showed that for a $\mathrm{VCl}_{3}$ concentration of $0.017 \mathrm{~mol} \mathrm{~L}^{-1}$ (Schnetger and Lehners recipe) ${ }^{16}$ at

$31530^{\circ} \mathrm{C}, 100 \%$ of nitrate was reduced after $2 \mathrm{~h}$ and only $45 \mathrm{~min}$ was needed with $0.07 \mathrm{~mol} \mathrm{~L}^{-1}$.

316 Therefore it is possible to apply this nitrate reduction technique to 2D DET nitrate imagery 
317 accepting a relaxation effect during $45 \mathrm{~min} i$. e. of about roughly $2 \mathrm{~mm}$ (in all directions) 318 which is not ideal for a sub-millimeter resolution image.

\subsubsection{Hyperspectral analysis: accuracy and LOD improvements}

Use of the vanadium chloride solution (a dark green solution) creates a second problem as the solution absorbs a certain quantity of light with a particular spectral signature and therefore is

322 likely to raise the LOD of the 2D DET nitrate imagery. Preliminary tests performed with commercial flatbed scanner showed that $\mathrm{VCl}_{3}$ (at $0.07 \mathrm{~mol} \mathrm{~L} \mathrm{~L}^{-1}$ ) interferences prevent the detection of nitrate. Further investigation revealed that the hyperspectral camera allows detecting nitrate (despite $0.07 \mathrm{~mol} \mathrm{~L}^{-1}$ of $\mathrm{VCl}_{3}$ ) but only above $10 \mu \mathrm{mol} \mathrm{L} \mathrm{L}^{-1}$. As shown in the spectra described in the supporting information (SI-3), accuracy of nitrite detection is constrained by the absorption band of the complex coloring reagent + probe + reducing reagent gels between $450 \mathrm{~nm}$ and $590 \mathrm{~nm}$. However, the adjunction of a dark green vanadium chloride gel considerably modifies the background. The signal brought by vanadium(III) (between 490 and $660 \mathrm{~nm}$ ) is spread over the entire visible spectrum including where the nitrite peak develops. To overcome this limitation and keep acceptable reduction kinetics we chose to work with a final concentration of $\mathrm{VCl}_{3}=0.035 \mathrm{~mol} \mathrm{~L}^{-1}$ in the reducing reagent gel, with a time span for nitrate reduction of $20 \mathrm{~min}$ at the temperature of $50^{\circ} \mathrm{C}$.

Standard curves obtained for nitrite and nitrate (SI-4) with the optimized protocol for an assemblage of coloring reagent + calibration+reducing reagent gels show good linearity $\left(\mathrm{r}^{2}=\right.$ 0.996 and 0.989 for nitrite and nitrate respectively) with a LOD about $1.7 \mu \mathrm{mol} \mathrm{L}^{-1}$ (3-fold standard deviation of the blank reflectance), a $5 \mu \mathrm{mol} \mathrm{L}^{-1}$ LOQ and a $2 \mu \mathrm{mol} \mathrm{L}{ }^{-1}$ accuracy. By analogy with length of cuvettes, thickening the probe gel increases reflectance and therefore would allow LOD increasing if necessary. However, a thicker gel requires an increase of in situ deployment time and results in a decreasing of spatial definition of 2D structures because of lateral diffusion within the gel. ${ }^{25}$ 
342 The comparison of nitrite and nitrate standard curves allows the determination of an averaged 343 nitrate reduction efficiency by comparison of slopes. A $100 \%$ nitrate reduction rate would 344 lead to a slope nitrate $/ \mathrm{slope}_{\text {nitrite }}$ equal to 1 . Standard curve for nitrate has a slope of 0.0450 (SI-4) 345 while the standard curve for nitrite has a slope of 0.0501 ; the ratio indicates a nitrate reduction 346 efficiency of about $90 \%$. Once, the quantification of nitrate reduction efficiency determined, it 347 is possible to quantify the nitrate concentration within the gel despite the lack of a total 348 reduction of nitrate. Another experiment was realized with slightly different conditions 349 (reduction time of 10 minutes and temperature of $40^{\circ} \mathrm{C}$ ) that gave a nitrate reduction 350 efficiency of about $75 \%$. These results suggest that the interplay between nitrate reduction 351 kinetics and diffusion smoothing of 2D structures has to be considered carefully while 352 biogeochemical interpretations about microniches or other sedimentary structures are 353 hypothesized. A simple transport modeling shows that if only diffusion governs nitrate 354 transportation within a gel, a production layer will vertically spread over twice its thickness 355 after $40 \mathrm{mn}$ at $50^{\circ} \mathrm{C}$ (see details in SI-5). This means that a very precise knowledge about 356 diffusion of chemical species within the gel at different temperatures and for each step of the 357 colorimetric protocol has to be acquired for good modeling of signal relaxation and therefore 358 2D structure reconstruction. A more empiric approach was chosen to evaluate such relaxation 359 in the section 3.1.1 for nitrite. Results suggest that, once the pink azo dye is formed, diffusion 360 is greatly slowed down and cannot be observed within the time of the experiment. During the 361 second step of the protocol (nitrate reduction using $\mathrm{VCl}_{3}$ ), nitrate can diffuse and signal be 362 relaxed since the Griess reaction did not occur yet. Our results suggest that at a millimeter 363 scale, this would not significantly affect structure shapes but probably affect more chemical 364 gradients across their edges. 


\subsubsection{Application on an incubated sediment}

366 Unlike the nitrite testing (section 3.1), this experiment used sediment unsieved and non-

367 homogenized in order to keep potential sedimentary or biogenic structures. Figure 3 shows 368 the distribution of nitrite obtained from the first scan (i.e. first reagent gel over the probe gel)

369 in the left panel and nitrite + reduced nitrate from the second scan in the central panel.

370 Efficiency of nitrate reduction was taken as $90 \%$ according to the standardization. The right

371 panel shows the resulting nitrate distribution after nitrite subtraction and efficiency correction.

372 Nitrite remains below $5 \mu \mathrm{mol} \mathrm{L} \mathrm{L}^{-1}$ over the $15 \mathrm{~cm}$ of the gel inserted into the sediment while

373 nitrate shows maximal concentrations over $40 \mu \mathrm{mol} \mathrm{L} \mathrm{L}^{-1}$ near the SWI. Despite lateral

374 heterogeneity, nitrate seems to decrease below the LOD at $4 \mathrm{~cm}$ depth. One can note a

375 spherical patch about $13 \mathrm{~cm}$ below the SWI.

376

377 Figure 4 and Figure 5 show separately nitrite and nitrate 2D distribution respectively 378 extracted from Figure 3 with appropriate rescaling. Vertical lines over the picture correspond 379 to profile extractions shown in right panels. The three nitrite profiles are very similar 380 suggesting little lateral variability. Near the SWI, nitrite concentration is about $6 \mu \mathrm{mol} \mathrm{L}{ }^{-1}$.

381 There is a decrease to a minimum of $\sim 2 \mu \mathrm{mol} \mathrm{L} \mathrm{L}^{-1}$ at $4 \mathrm{~cm}$ depth. Below, nitrite concentration

382 remains roughly constant between LOD and LOQ (limit of quantification). Nitrate profiles

383 show a more complex pattern (Figure 5). All profiles show a nitrate maximum approximately

$3841 \mathrm{~cm}$ below the SWI indicating nitrification. ${ }^{26,28}$ Then nitrate reaches zero at $5 \mathrm{~cm}$ depth. 385 Below $10 \mathrm{~cm}$ depth, nitrate seems to be stable at a concentration below LOD. The variability 386 of maximum intensities that ranges between 20 and $40 \mu \mathrm{mol} \mathrm{L}^{-1}$ illustrates the high lateral 387 variability of such sediment at a millimeter scale. Such a variability is visible as well for 388 dissolved iron and phosphorus for in situ deployments in the same area ${ }^{13}$ mostly explained by 389 the presence of active burrows of polychaetes. 
390 The lateral variability of nitrate gradients intensity below the SWI highlights the importance

391 of bioturbation for nitrogen cycling ${ }^{29-32}$ and especially for oxidation of ammonium and

392 nitrate release from sediment into water column. Bioturbation can enhance nitrate

393 consumption producing $\mathrm{N}_{2} \mathrm{O}$ and/or $\mathrm{N}_{2}$ by denitrification or by oxydation of reduced

394 compounds such as $\mathrm{FeS}$ or $\mathrm{Fe}^{2+}, 33$ or producing again ammonium by dissimilatory nitrate

395 reduction to ammonium (DNRA). This is of major importance considering the fact that

396 nutrient availability controls benthic primary production and that microphytobenthic mats

397 play an important role on the food web and on stabilization of the sedimentary substratum. ${ }^{34}$

398 Important nitrate production would also imply reduction of oxidizers such as manganese and

399 iron oxy(hydroxi)des and therefore enhancement of metal remobilization and recycling. ${ }^{33,35,36}$

400 Therefore, we offer here the possibility to examine in 2 dimensions, the spatial nitrite and

401 nitrate variability at a sufficient resolution that allows to describe chemical gradients

402 generated by a mm-sized dead organism, a root apex, along a burrow wall. Further, these

403 processes could be quantified with appropriate modeling. ${ }^{37,38}$ Indeed, a double layer gel can

404 be performed to sample at the same location both nitrite/nitrate and iron/phosphate couples.

405 The image of nitrate distribution shows microenvironments at subsurface, between the oxic

406 zone and the iron remobilization zone, and more surprisingly $13 \mathrm{~cm}$ below the interface

407 suggesting that noisy profiles in the literature, that are often obtained from core slicing could

408 not be artifacts but could be a result of the sampling of a microenvironment similar to the one

409 shown here that peaks at about $30 \mu \mathrm{mol} \mathrm{L}^{-1}$.

410 Combining colorimetry, gel sampling and hyperspectral imagery allows assessing, at sub-

411 millimeter resolution in 2 dimensions, nitrite and nitrate production/consumption hotspots

412 within a range between 1 and $40 \mu \mathrm{mol} \mathrm{L}^{-1}$ without sampling discretization. However, due to

413 relaxation effects, fidelity of concentration is achieved at a millimeter resolution. This

414 technique is a good alternative to microsensors that allow sub-millimeter profiling but are

415 difficult to perform and time-consuming as several profiles are needed to assess 2D 
416 distribution (at a low resolution anyway). The protocol proposed here allowed to describe

417 nitrate release as microniches below the "zero-nitrate" layer and to evidence the lateral

418 variability of nitrate concentration in estuarine muddy sediment. Such technique offers

419 numerous perspectives for laboratory and in situ studies dealing with the reactivity of

420 microenvironments such as burrows or decaying macro(meio)fauna locations. Combined to

421 other high-resolution $2 \mathrm{D}$ analyses such as $\mathrm{Fe} / \mathrm{PO}_{4}$ gels, this method will provide valuable

422 insights on the mechanisms that control nutrient release and primary production feedback.

\section{Acknowledgements}

424 This study is part of the RS2E - OSUNA project funded by the Région Pays de la Loire.

425 Thanks to Romain Levrard, Livia Defaye and Manuel Giraud for their technical help.

427 Supporting information

428 Supporting information shows a scheme of the probe gel (SI-1); the kinetics of nitrate

429 reduction into nitrite as a function of vanadium chloride concentration (SI-2); different spectra

430 of reflectance obtained with a hyperspectral camera for the gels at different stages of

431 processing (SI-3); standard curves for nitrite and nitrate that allows to quantify reducing

432 efficiency fo nitrate into nitrite (SI-4); and the theoretical relaxation of a nitrate peak after gel

433 processing considering molecular diffusion (SI-5). This information is available free of charge

434 via the Internet at http://pubs.acs.org/.

435 
438 Figure 1: kinetics of nitrite colorimetric reaction on a gel. A: color evolution of the standard 439 curve over time. B: extracted reflectance (green channel) from images. C: overtime profile

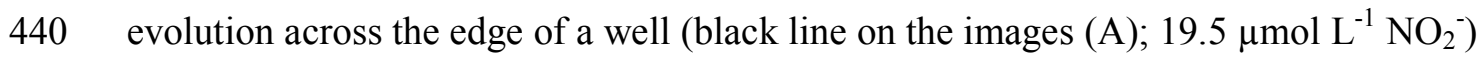

441 Figure 2: 2D nitrite concentration distribution and examples of selected profiles for a sieved 442 and homogenized sediment of the Brillantes mudflat Loire estuary, artificially "bioturbated" 443 by a $3 \mathrm{~cm}$-diameter tube. Dots are data and lines are smoothed profiles.

444 Figure 3: 2D nitrite (left), nitrite + reduced nitrate (middle), calculated nitrate (right) for an 445 incubated sediment from the Brillantes mudflat, Loire estuary

446 Figure 4: 2D nitrite distribution (left) extracted from Figure 3 and rescaled, and examples of 447 selected profiles for incubated sediment of the Brillantes mudflat, Loire estuary. Dots are data 448 and lines smoothed profiles.

449 Figure 5: 2D nitrate distribution (left) extracted from Figure 3, and examples of selected 450 profiles for an incubated sediment of the Brillantes mudflat, Loire estuary. Dots are data and 451 lines smoothed profiles. 
453

454

455

456

457

458

459
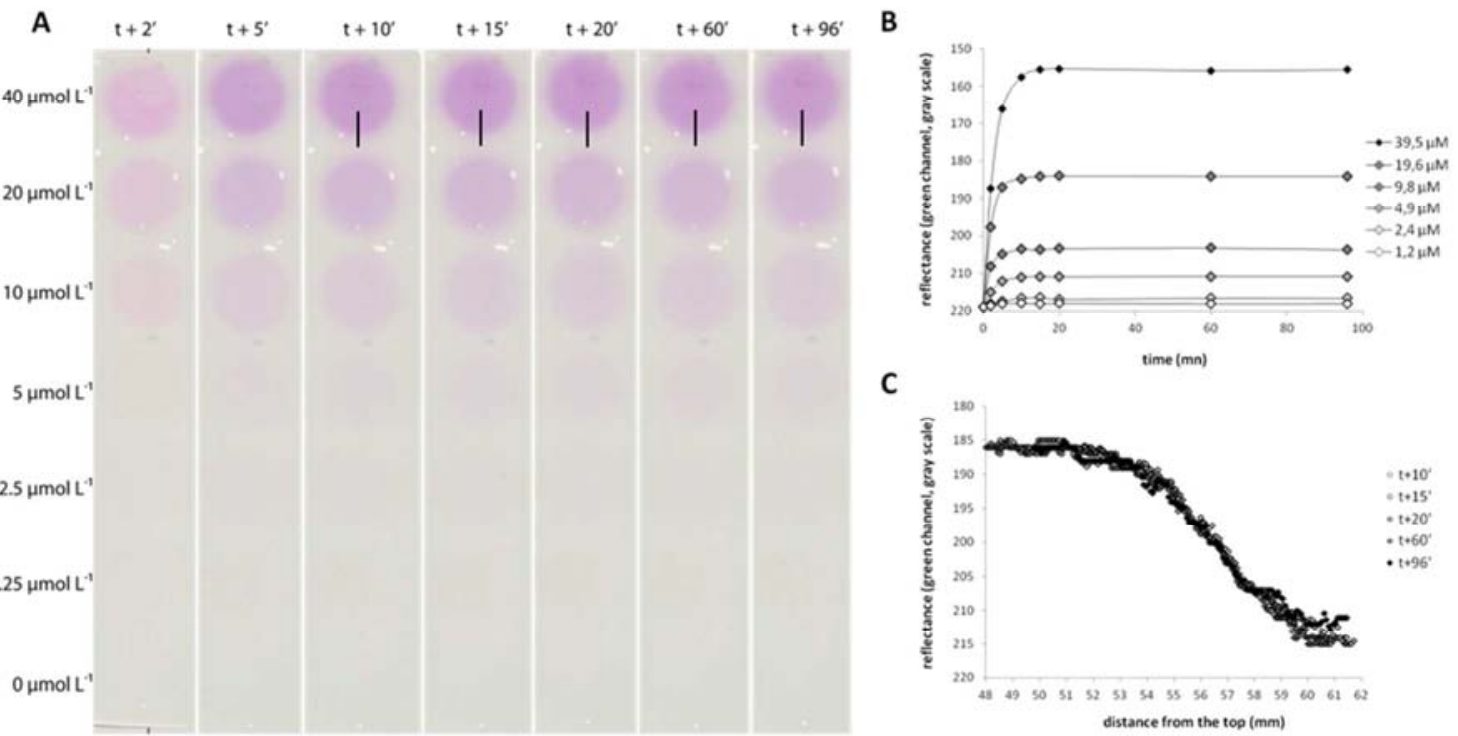

\section{C}

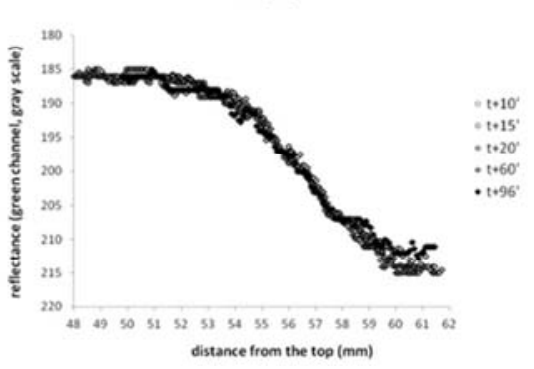

Figure 1: kinetics of nitrite colorimetric reaction on a gel. A: color evolution of the standard curve over time. B: extracted reflectance (green channel) from images. C: overtime profile evolution across the edge of a well (black line

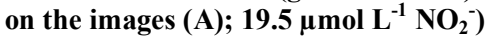



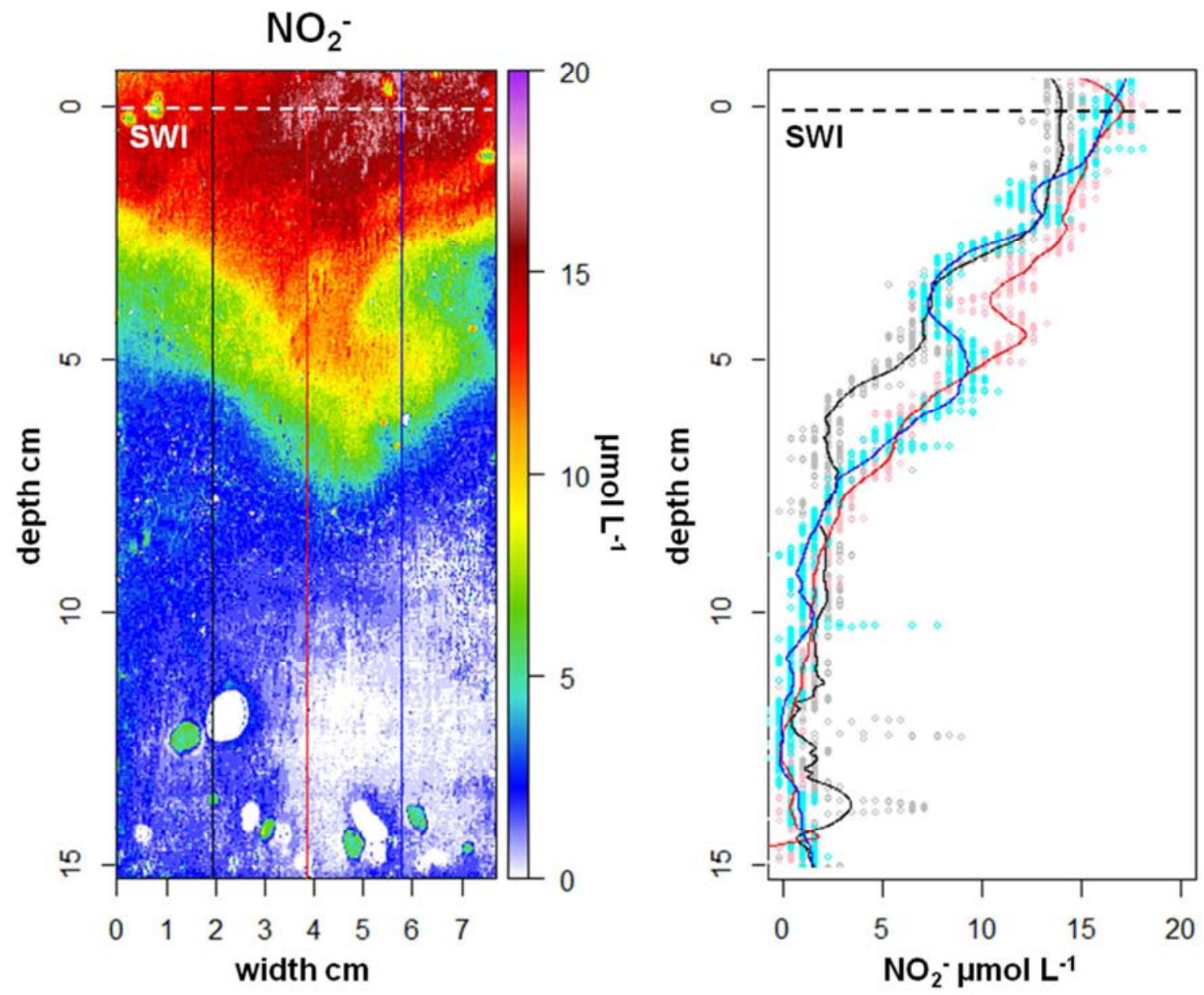

462

Figure 2: 2D nitrite concentration distribution and examples of selected profiles for a sieved and homogenized 464 sediment of the Brillantes mudflat Loire estuary, artificially "bioturbated" by a 3 cm-diameter tube. Dots are data 465 and lines are smoothed profiles. 

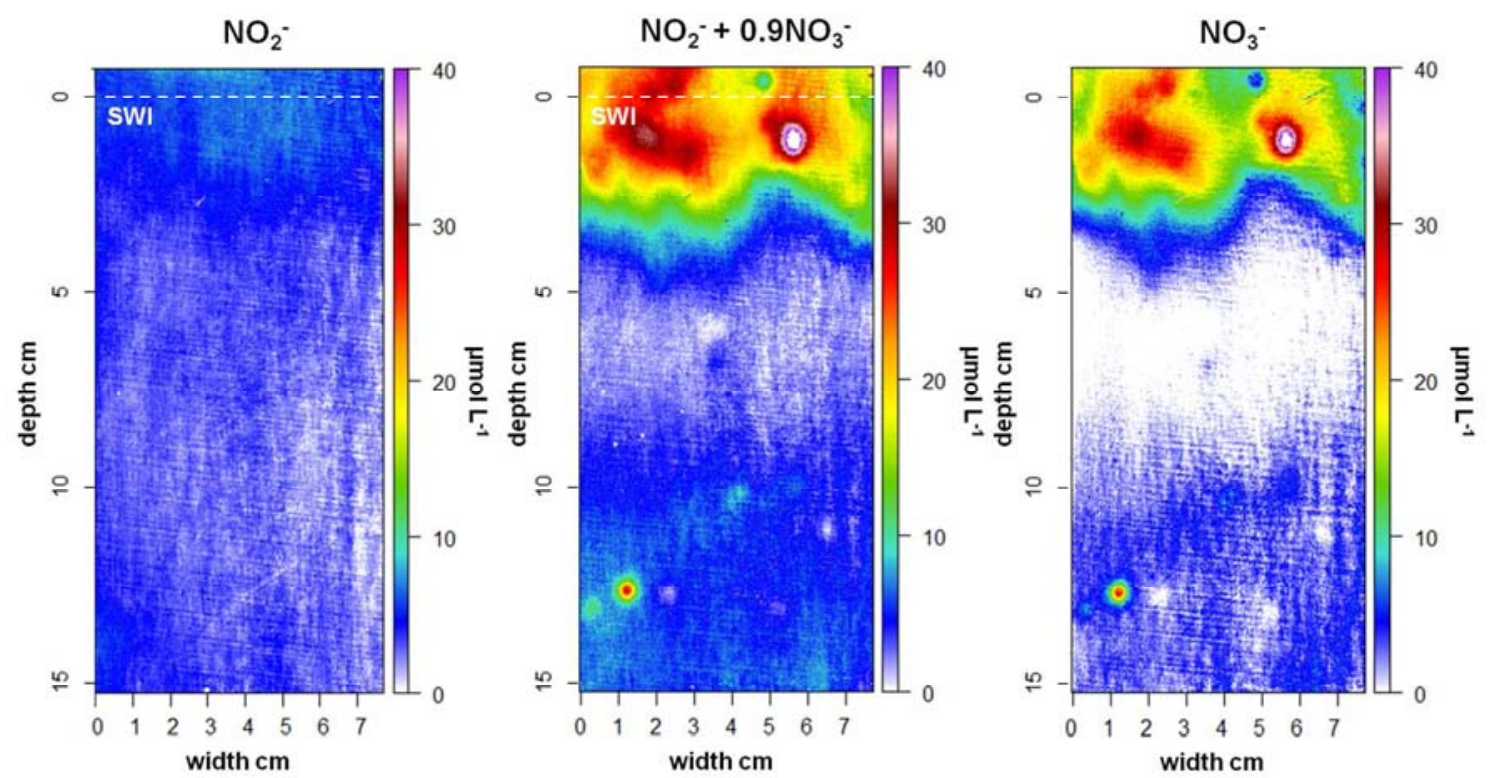

Figure 3: 2D nitrite (left), nitrite + reduced nitrate (middle), calculated nitrate (right) for an incubated sediment from 471 the Brillantes mudflat, Loire estuary 

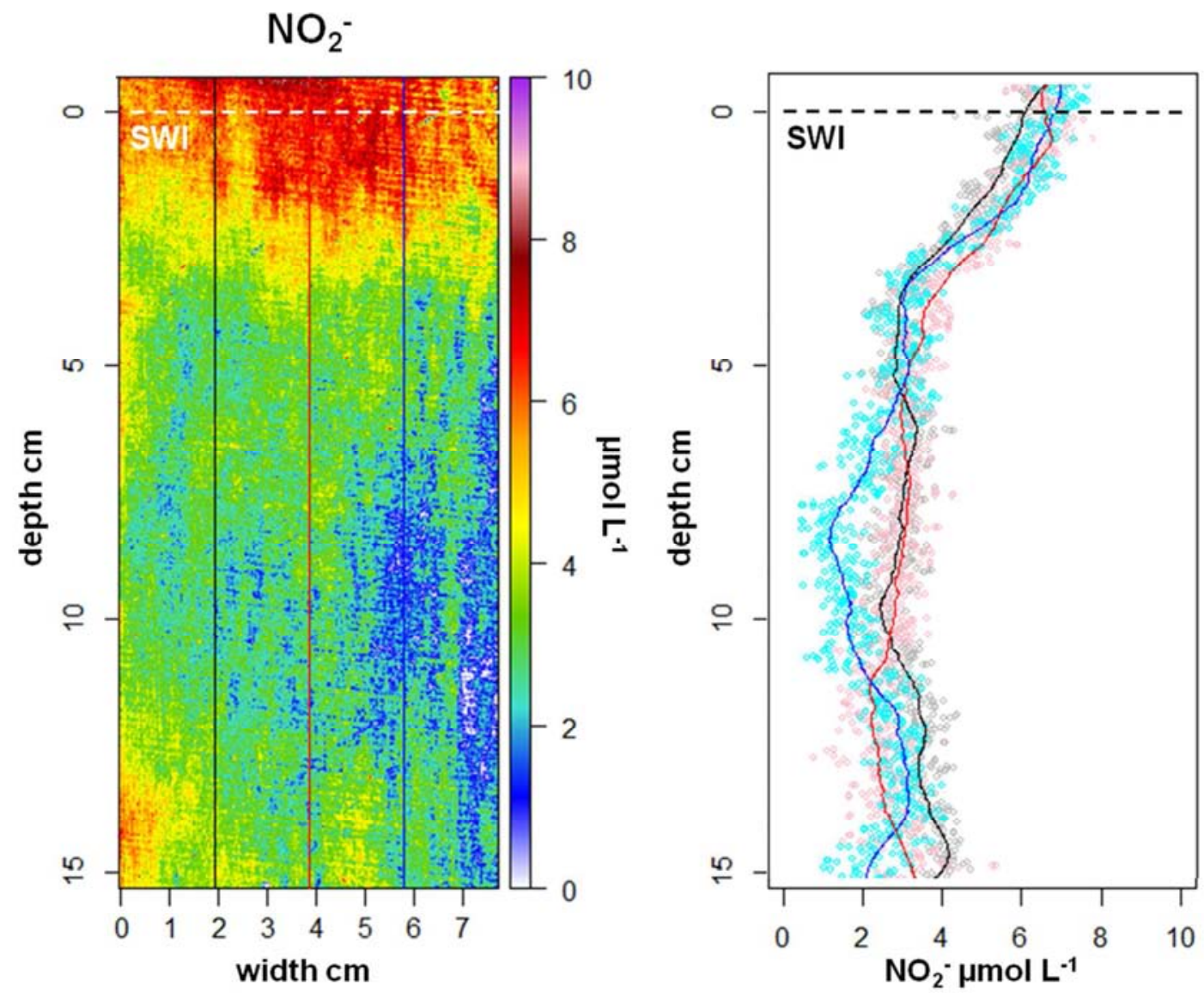

473

Figure 4: 2D nitrite distribution (left) extracted from Figure 3 and rescaled, and examples of selected profiles for incubated sediment of the Brillantes mudflat, Loire estuary. Dots are data and lines smoothed profiles.

476

477 


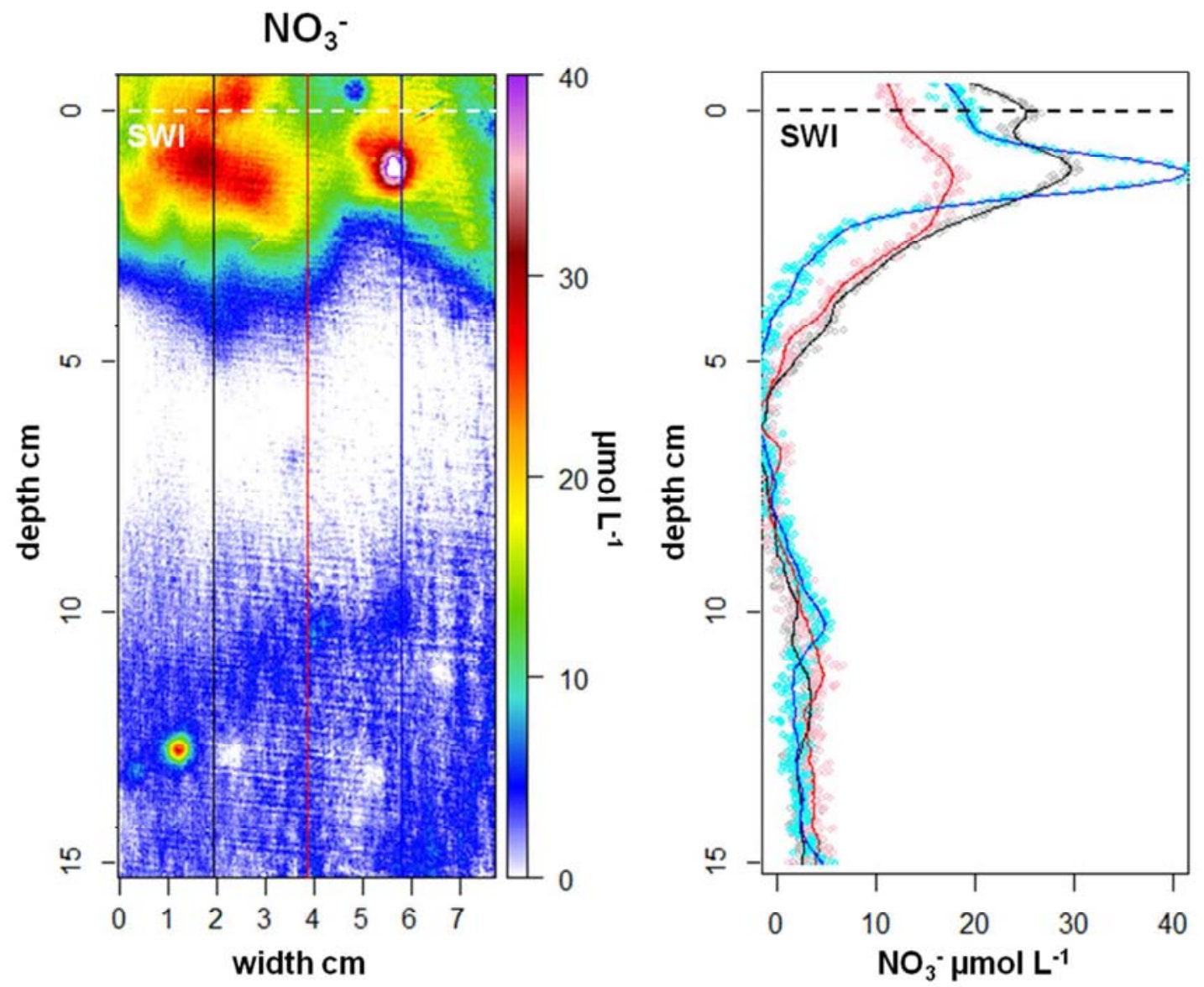

Figure 5: 2D nitrate distribution (left) extracted from Figure 3, and examples of selected profiles for an incubated sediment of the Brillantes mudflat, Loire estuary. Dots are data and lines smoothed profiles. 


\section{References}

485

486

487

488

489

490

491

492

493

494

495

496

497

498

499

500

501

502

503

504

505

506

507

508

509

510

511

512

513

514

515

516

517

518

519

520

521

522

523

524

525

526

527

528

529

530

531

532

533

534
(1) Davison, W.; Grime, G. W.; Morgan, J. a. W.; Clarke, K. Distribution of dissolved iron in sediment pore waters at submillimetre resolution. Nature 1991, 352 (6333), 323 325.

(2) Monbet, P.; McKelvie, I. D.; Worsfold, P. J. Combined Gel Probes for the In Situ Determination of Dissolved Reactive Phosphorus in Porewaters and Characterization of Sediment Reactivity. Environ. Sci. Technol. 2008, 42 (14), 5112-5117.

(3) Koschorreck, M.; Brookland, I.; Matthias, A. Biogeochemistry of the sediment-water interface in the littoral of an acidic mining lake studied with microsensors and gelprobes. J. Exp. Mar. Biol. Ecol. 2003, 285-286, 71-84.

(4) Metzger, E.; Viollier, E.; Simonucci, C.; Prévot, F.; Langlet, D.; Jézéquel, D. Millimeter-scale alkalinity measurement in marine sediment using DET probes and colorimetric determination. Water Res. 2013, 47 (15), 5575-5583.

(5) Stockdale, A.; Davison, W.; Zhang, H. Micro-scale biogeochemical heterogeneity in sediments: A review of available technology and observed evidence. Earth-Sci. Rev. 2009, 92 (1-2), 81-97.

(6) Shuttleworth, S. M.; Davison, W.; Hamilton-Taylor, J. Two-Dimensional and Fine Structure in the Concentrations of Iron and Manganese in Sediment Pore-Waters. Environ. Sci. Technol. 1999, 33 (23), 4169-4175.

(7) Krause, S.; Tecklenburg, C.; Munz, M.; Naden, E. Streambed nitrogen cycling beyond the hyporheic zone: Flow controls on horizontal patterns and depth distribution of nitrate and dissolved oxygen in the upwelling groundwater of a lowland river. $J$. Geophys. Res. Biogeosciences 2013, 118 (1), 54-67.

(8) Mortimer, R. J. G.; Krom, M. D.; Harris, S. J.; Hayes, P. J.; Davies, I. M.; Davison, W.; Zhang, H. Evidence for suboxic nitrification in recent marine sediments. Mar. Ecol. Prog. Ser. 2002, 236 (1), 31-35.

(9) Jézéquel, D.; Brayner, R.; Metzger, E.; Viollier, E.; Prévot, F.; Fiévet, F. Twodimensional determination of dissolved iron and sulfur species in marine sediment pore-waters by thin-film based imaging. Thau lagoon (France). Estuar. Coast. Shelf Sci. 2007, 72 (3), 420-431.

(10) Robertson, D.; Teasdale, P. R.; Welsh, D. T. A novel gel-based technique for the high resolution, two-dimensional determination of iron (II) and sulfide in sediment. Limnol. Oceanogr. Methods 2008, 6 (10), 502-512.

(11) Pagès, A.; Teasdale, P. R.; Robertson, D.; Bennett, W. W.; Schäfer, J.; Welsh, D. T. Representative measurement of two-dimensional reactive phosphate distributions and co-distributed iron(II) and sulfide in seagrass sediment porewaters. Chemosphere 2011, $85(8), 1256-1261$.

(12) Cesbron, F.; Metzger, E.; Launeau, P.; Deflandre, B.; Delgard, M.-L.; Thibault de Chanvalon, A.; Geslin, E.; Anschutz, P.; Jézéquel, D. Simultaneous 2D Imaging of Dissolved Iron and Reactive Phosphorus in Sediment Porewaters by Thin-Film and Hyperspectral Methods. Environ. Sci. Technol. 2014, 48 (5), 2816-2826.

(13) Thibault de Chanvalon, A.; Metzger, E.; Mouret, A.; Cesbron, F.; Knoery, J.; Rozuel, E.; Launeau, P.; Nardelli, M. P.; Jorissen, F. J.; Geslin, E. Two-dimensional distribution of living benthic foraminifera in anoxic sediment layers of an estuarine mudflat (Loire estuary, France). Biogeosciences 2015, 12 (20), 6219-6234.

(14) Larsen, L. H.; Damgaard, L. R.; Kjær, T.; Stenstrøm, T.; Lynggaard-Jensen, A.; Revsbech, N. P. Fast responding biosensor for on-line determination of nitrate/nitrite in activated sludge. Water Res. 2000, 34 (9), 2463-2468.

(15) Revsbech, N. P.; Glud, R. N. Biosensor for laboratory and lander-based analysis of benthic nitrate plus nitrite distribution in marine environments. Limnol. Oceanogr. Methods 2009, 7 (11), 761-770. 
(16) Schnetger, B.; Lehners, C. Determination of nitrate plus nitrite in small volume marine water samples using vanadium(III)chloride as a reduction agent. Mar. Chem. 2014, 160, 91-98.

(17) García-Robledo, E.; Corzo, A.; Papaspyrou, S. A fast and direct spectrophotometric method for the sequential determination of nitrate and nitrite at low concentrations in small volumes. Mar. Chem. 2014, 162, 30-36.

(18) Griess, P. Bemerkungen zu der Abhandlung der HH. Weselsky und Benedikt „Ueber einige Azoverbindungen" $\square$. Berichte Dtsch. Chem. Ges. 1879, 12 (1), 426-428.

(19) Bratton, A. C.; Marshall, E. K.; Hendrickson, W. the technical assistance of D. B. and A. R. A New Coupling Component for Sulfanilamide Determination. J. Biol. Chem. 1939, $128(2), 537-550$.

(20) Zhang, H.; Davison, W. Diffusional characteristics of hydrogels used in DGT and DET techniques. Anal. Chim. Acta 1999, 398 (2), 329-340.

(21) Benyoucef, I.; Blandin, E.; Lerouxel, A.; Jesus, B.; Rosa, P.; Méléder, V.; Launeau, P.; Barillé, L. Microphytobenthos interannual variations in a north-European estuary (Loire estuary, France) detected by visible-infrared multispectral remote sensing. Estuar. Coast. Shelf Sci. 2014, 136, 43-52.

(22) Bendschneider, K.; R J Robinson. A new spectrophotometric method for determination of nitrite in sea water. J. Mar. Res. 1952, 11, 87-96.

(23) Strickland, J. D. H.; Parsons, T. R. A practical handbook of seawater analysis; Fisheries Research Board of Canada, 1972.

(24) Grasshoff, K.; Kremling, K.; Ehrhardt, M. Methods of Seawater Analysis; John Wiley \& Sons, 2009.

(25) Harper, M. P.; Davison, W.; Tych, W. Temporal, Spatial, and Resolution Constraints for in Situ Sampling Devices Using Diffusional Equilibration: Dialysis and DET. Environ. Sci. Technol. 1997, 31 (11), 3110-3119.

(26) Burgin, A. J.; Hamilton, S. K. Have we overemphasized the role of denitrification in aquatic ecosystems? A review of nitrate removal pathways. Front. Ecol. Environ. 2007, $5(2), 89-96$.

(27) Wood, E. D.; Armstrong, F. a. J.; Richards, F. A. Determination of nitrate in sea water by cadmium-copper reduction to nitrite. J. Mar. Biol. Assoc. U. K. 1967, 47 (1), 23-31.

(28) Sørensen, J.; Jørgensen, B. B.; Revsbech, N. P. A comparison of oxygen, nitrate, and sulfate respiration in coastal marine sediments. Microb. Ecol. 1979, 5 (2), 105-115.

(29) Aller, R. C.; Aller, J. Y. The effect of biogenic irrigation intensity and solute exchange on diagenetic reaction rates in marine sediments. J. Mar. Res. 1998, 56 (4), 905-936.

(30) Bonaglia, S.; Nascimento, F. J. A.; Bartoli, M.; Klawonn, I.; Brüchert, V. Meiofauna increases bacterial denitrification in marine sediments. Nat. Commun. 2014, 5, 5133.

(31) Gilbert, F.; Aller, R. C.; Hulth, S. The influence of macrofaunal burrow spacing and diffusive scaling on sedimentary nitrification and denitrification: An experimental simulation and model approach. J. Mar. Res. 2003, 61 (1), 101-125.

(32) Stief, P. Stimulation of microbial nitrogen cycling in aquatic ecosystems by benthic macrofauna: mechanisms and environmental implications. Biogeosciences 2013, 10 (12), 7829-7846.

(33) Hulth, S.; Aller, R. C.; Gilbert, F. Coupled anoxic nitrification/manganese reduction in marine sediments. Geochim. Cosmochim. Acta 1999, 63 (1), 49-66.

(34) MacIntyre, H. L.; Geider, R. J.; Miller, D. C. Microphytobenthos: The ecological role of the "secret garden" of unvegetated, shallow-water marine habitats. I. Distribution, abundance and primary production. Estuaries 1996, 19 (2), 186-201.

(35) Anschutz, P.; Zhong, S.; Sundby, B.; Mucci, A.; Gobeil, C. Burial efficiency of phosphorus and the geochemistry of iron in continental margin sediments. Limnol. Oceanogr. 1998, 43 (1), 53-64.

(36) Fernandes, S. O.; Javanaud, C.; Aigle, A.; Michotey, V. D.; Guasco, S.; Deborde, J.; Deflandre, B.; Anschutz, P.; Bonin, P. C. Anaerobic nitrification-denitrification mediated by Mn-oxides in meso-tidal sediments: Implications for $\mathrm{N} 2$ and $\mathrm{N} 2 \mathrm{O}$ production. J. Mar. Syst. 2015, 144, 1-8.

(37) Zhu, Q.; Aller, R. C.; Fan, Y. Two-dimensional pH distributions and dynamics in bioturbated marine sediments. Geochim. Cosmochim. Acta 2006, 70 (19), 4933-4949. 
592 (38) Thibault de Chanvalon, A.; Metzger, E.; Mouret, A.; Geslin, E.; Knoery, J.; Meysman, 593

594

595 F. J. R. Two dimensional mapping of iron release in marine sediments at submillimetre scale. Mar. Chem. in press. 